\title{
STRATÉGIE DE COMMUNICATION ENVIRONNEMENTALE ET CONSTRUCTION DE LÉGITIMITÉ Le cas de PSA Peugeot Citroën
}

\author{
ALAIN DEBENEDETTI ET DÉBORAH PHILIPPE
}

Alain DEBENEDETTI, Mâtre de Conférences à l'Université Paris-Est. IRG. Il enseigne le marketing et ses recherches se focalisent sur le comportement du consommateur, notamment les aspects symboliques et non marhands de la consommation. Conta't : Alain.Debenedetti@univ-mlv:fr

Déborah PHILIPPE. Professeure Assistante en Stratégie à la Faculté des HEC de l'Université de Lausanne. Ses recherches s'inscrivent dans le champ de la sociologie des organisations et portent sur les stratégies mises en oeuvre par les organisations pour gérer leurs évaluations sociales externes (statut. légitimité. ét répuatation).

Contact : deborah.philippe@unil.ch

Les auteurs interessés par une sounission pour la rubrique "Stratégies" doivent prendre contact avec François Courvoisier (Franois.Courvoisier@ he-arc.ch) pour toute proposition. Le format suit des règles spécifiques : vous pouvez vous référer aux instructions aux auteurs simuées à la fin de ce muméro.

\section{Résumé}

Cet article propose un éclairage sur les stratégies de commanication corporate, et en particulier sur les stratégies emplovées par les marques pour aligner le contenu de leur communication avec les attentes de leurs cibles (clients, employés, actionnaires etc:). En illustration, nous montrons comment PSA Peugeot Citrö̈n cherche à légitimer son action environnementale auprès de ses différentes parties prenantes, en s'appavant sur trois formes de légitimité (pragmatique. cognitive et morale) déclinées én fonction de l'audience ciblée.

Mots-clés : Communication environnementale, Légitimité. Corporate marketing. Industrie automobile

\section{Abstract}

ENVIRONMENTAL COMMUNICATION STRATEGY AND CONSTRUCTION OF LEGITIMACY. THE CASE OF PSA PEUGEOT CITROËN.

This article aims at enlightening corporate communication strategies, with a specific focts on the strategies that brands implement to align the content of their communication with their multiple audiences' expectations customers, employees, shareholders etc.). To illustrate these strategies, we show how PSA Peugeot Citroën is attempting to legitimate its environmental actions towards its multiple stakeholders. building on three form. of legitimacy (pragmatic, cognitive, and moral) that are adapted to each targeted audience

Key Words: Environmental communication, Legitimacy, Corporate marketing. Automobile industry 


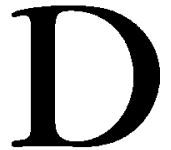

epuis 2008 , le rapport annuel de PSA Peugeot Citroën (noté PSA par la suite) s"intitule "Rapport d'Activité et de Développement Durable ", et souligne, dès la page de garde, à grand renfort de coulcur verte et de formes végétales (11), la volonté du groupe d'être perçu comme impli qué dans la préservation de l'environnement. Ce choix reflete la pression institutionnelle dans laquelle l'industric automobile évolue aujourd'hui : on n'attend plus seulement d'un constructeur qu il produise des voitures sûres, il doit également produire des voitures propres. Or, l'inclustrie automobile représente aujourd'hui $70 \%$ des émissions de gaz polluants liés aux transports en Europe. Légitimer son action environnementale constitue un véritable défi, qui pour être relevé, nécessite de bâtir une stratégie de communication environnementale adécquate. Dans cette optique, le message clélivré doit correspondre aux attentes précises et parfois divergentes de chaque partie prenante du secteur (consommateurs, actionnaires, employés, etc.) et la convaincre de l'engagement environnemental du constructeur. Dans cet article, nous nous intéressons à PSA, 2 constructeur curopéen, en raison de sa réputation de leader environnemental dans l'industrie alutomobile. Apress avoir montré le lien entre corporate marketing et légitimité, nous présenterons, au travers de l'exemple de PSA', les différents types de strattégies de légitimation environnementale et les formes que ces stratégies petvent revètir dans le contexte de l'industrie automobile.

\section{CORPORATE MARKETING ET LEGITIMITE}

Le corporate marketing est une terminologic ombrelle qui regroupe plusieurs concepts (ex : marque corporate, communication corporate, identité corporate etc.) cui se trouvent depuis longtemps au coeur des problématiques marketing des entreprises mais qui sont ici appliqués à un niveau institutionnel (1). À travers sa dimension communication, le corporate marketing désigne donc les actions qu'une entreprise déploie pour délivrer des informations sur ses valeurs et ses missions (1), satns nécessalirement faire référence à une marque développée par l'entreprise ou un produit vendu. Sa principale caractéristique est de ne pas uniquement s'adresser au consommateur final, mais de cibler l'ensemble des parties prenantes intencs (cmployés) ou externes (fournisseurs, clients, actionnatires, etc.) de lentreprise. Lun des principaux objectifs du corporate marketing est de construire et de gérer l'image de la marque à un niveau institutionnel. Pour cela, lentreprise délive des informations qui correspondent à la logicue institutionnelle en vigueur (ici, le besoin d'être responsable vis-à-vis de l'environnement). Elle peut également communiquer des informations qui répondent aux intérêts, aux croyances et aux valeurs spécificues des différentes cibles (ici, légitimer le comportement environnemental de l'entreprise vis-a-vis de ses parties prenantes). Pour construire et défendre son image au niveau institutionnel, une organisation comme PSA doit done entretenir des relations avec de multiples parties prenantes (7). Autrement dit, il s'agit d'être compris et approuvé par l'ensemble des cibles qui, a leur manière. consomment "la marque, en déployant pour chacune de ces cibles, des symboles évocateurs forts (12) Chaque cible étant susceptible d'avoir des attentes différentes, il est donc nécessaire, dans le contexte de PSA de légitimer ses actions environnementales auprès de ses publics cibles en clélivrant un message qui tienne compte de leur systeme de valeurs.

Au traters de la dimension communication de son marketing corporate, PSA cherche ainsi à élaborer une stratégie discursive consistant à expliquer et à rationnaliser ses actions environnementales. Le résultat recherché est d'influencer la manière dont la cible va percevoir la réalité de l'organisation (6) et ainsi construire narrativement sá légitimité organisationnelle autour du thème du respect de l'environnement

La recherche de légitimité s'inscrit de plus en plus dans la stratégie marketing des organisations (3). La légitimité se définit comme "la perception généralisée ou supposition selon laquelle les actions d'une entité sont désirables, et appropriées au sein d'un système socialement construit de normes, valeurs, croyances et définitions " (12). Elle revêt plusieurs formes - pragmatique, morale et cognitive - (12) qui impliquent toutes que les activités de l'organisation (PSA dans notre exemple) soient socialement désirables et appropriées. Cependant, chacune d'entre elles possède une dynamique propre. La légitimité pragmatique repose sur l'intérêt procuré a la cible: laction est légitime dans la mesure où elle procure des bénéfices à l'audience ciblée. La légitimité morale repose sur la capacité de l'organisation à contribuer au bien être général. Au contraire de la légitimité pragmatique, qui repose sur l'évaluation de bénéfices individuels, la légitimité morale reflète la capacité de l'organisation à "faire le bien "au sein d'un système socialement construit de valeurs et croyances. Enfin. la légitimité cognitive est beaucoup moins explicite et reposc essentiellement sur l'acceptation d'une information présentée comme allant de soi.

\section{LÉGITIMITÉ PRAGMATIQUE : UNE PRIORITE DANS LES COMMUNICATIONS FINANCIÈRES ET ORIENTÉES CLIENTS ?}

Le contexte réglementaire actuel favorise l'utilisation d'un discours pragmatique sur l'environnement, en particulier à destination des clients finaux. En effet, début 2008 , le bonus écologique, qui récompense les acheteurs de véhicules propres et pénalise les acheteurs de véhicules polluants, a été mis en place. S'y est ajouté le super bonus (ou "prime à la calsse"), qui permet de bénéficier d'une prime de 1000 euros pour l'achat d'un véhicule non polluant à condition de retirer définitivement un véhicule de plus de 10 ans de la circulation. Dans ce contexte, la stratégie de légitimation pragmatique de PSA s'articule autour de plusieurs registres. 
Tableau 1

Exemples de stratégies de légitimation pragmatique de PSA

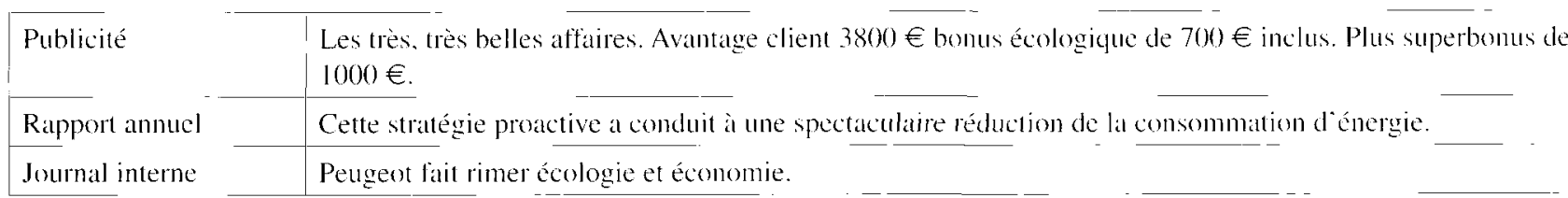

Tableau 2

Exemples de stratégies de légitimation morale de PSA

\begin{tabular}{|c|c|}
\hline Publicité & Réduisez volre empreinte carbone. \\
\hline Rapport annuel & $\begin{array}{l}\text { En Argentine. PSA Peugeot Citroën a réalisé plusieurs projets en } 2008 \text { avec Cascos Verdes, une organisation non } \\
\text { gouvernementale locale. A travers le programme "Conscience verte" nous aidons de jeunes handicapés mentaux à } \\
\text { augmenter leur chance d"aller à l'université et de jouer un rôle dans la société en les faisant participer a une forma- } \\
\text { tion de } 2 \text { ans sur la protection de l'environnement. }\end{array}$ \\
\hline Journal interne & $\begin{array}{l}\text { La mise en terre de ces plants enrichit un des espaces boisées prévu dans le projet environnemental de l usine el } \\
\text { contribue de ce fait à la reconstruction de la « Mara Atlantica ». }\end{array}$ \\
\hline
\end{tabular}

Lorsque la marque s'adresse à ses clients, elle met clairement en avant leur intelligence pratique, comme le montre par exemple le slogan " avec la prime BluEco, changez pour une voiture neuve, moins polluante ". Le consommateur " malin " préserve l'environnement et, par là même, profite d'une part de bonus gouvernementaux souvent abondés par la marque, et d'autre part économise de l'essence sans que cela nuise à son plaisir de conduire. Ia communication environnementale de PSA a destination des actionnaires fait de la préservation des ressources (en eau, électricité, etc.) un argument d'optimisation des couts, et prouve l'engagement du groupe en montrant qu'il respecte les normes environnementales en vigueur. La communication financière souligne également que l'engagement environnemental est source de profit, car il permet de bénéficier d'une meilleure position concurrenticlle. Le message délivré aux employés (qui sont souvent clients et pour un quatrt d'entre eux, actionnaires de l'entreprise) ne differe guère des deux précédents : PSA rappelle l'utilisation efficace des ressources naturelles pour construire des voitures agréables à conduire et économiques à l'usage.

La légitimité pragmatique semble adaptée à l'actionnaire en premier lieu, mais également au client, même si elle comporte un risque majeur : celui de réduire l'engagement environnemental individuel à une question purement monétaire. Le tableau 1 illustre la stratégie de légitimation pragmatique de PSA en donnant un exemple de communication pour chacune des parties prenantes évoquées.

\section{LÉGITIMITÉ MORALE : DE LA FIERTE DES EMPLOYÉS À LA CONSCIENCE ECOLOGIQUE DES CONSOMMATEURS?}

Les stratégies de communication reposant sur la légitimité morale semblent plus difficiles à décliner de manière originale (cf. tableau 2). Qu'elle sadresse aux employés ou aux consommateurs, la communication peut, par exemple, s'appuyer sur l'éducation aux questions environnementales en expliquant les principes de l'effot de serre ou en apprenant a la cible les gestes "éco-responsables". Le discours environnemental peut également souligner la participation à des programmes de recherche, ou le soutien à des organisations qui ceuvrent en faveur de l'environnement : par exemple, PSA participe à la construction d'un puits de carbone en Amćrique du Sud, et sponsorise des associations qui agissent au niveau local pour protéger l'environnement. La communication de PSA à destination des consommateurs souligne également les récompenses symboliques liées a l'acquisition d'un véhicule propre : cet achat procure en effet au consommateur le statut valorisant de défenseur de l'environnement. Il en est de méme pour les employés, que PSA cherche à rendre fiers en soulignant l'altruisme de sa démarche, et qui peuvent s'attendre à ce que le prestige issu de l'attitude responsable de PSA rejaillisse sur eux. Par ailleurs, communiquer sur les multiples efforts de PSA en termes de réduction des déponses d'énergic ou de matières premières (ex : quantité moyenne d'eau ou de peinture nécessaire à la fabrication d'un véhicule) dans une optique générale de réduction de l'empreinte environnementale du groupe est susceptible de contribuer à renforcer le sentiment dappartenance des cmployés

Les stratégies de légitimité morale contribuent à ce que collaborateurs et clients s'identifient à PSA. comme cela est souligné dans de nombreuses recherches sur la marque $(5,8)$. La recherche de légitimite morale, en revanche, ne constitue pas une priorité dans le discours délivré aux actionnaires, qui attendent logiquement en premier lieu une performance financière des actions environnementales de PSA. On peut cependant s'étonnor que la légitimité morale soit quasi absente des rapports annuels de PSA ciblant actionnaires et analystes financiers. En effet l'action environnementale ćtant sus- 
Tableau 3

Exemples de stratégies de légitimation cognitive de PSA

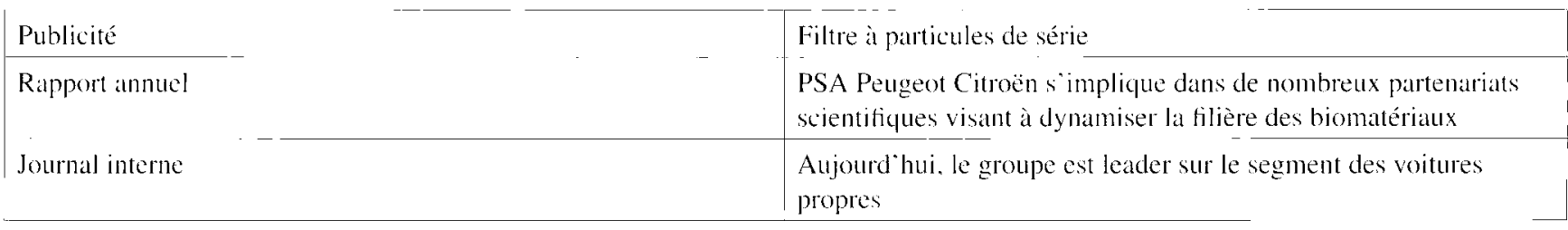

ceptible de jouer sur l'image de l'entreprise, elle peut donc indirectement accroitre le capital de la marque corporale, et contribuer à améliorer la productivité d'employés fiers d'appartenir à PSA. Ce manque d'utilisation de ce type de légitimation n'est pas propre a PSA. Une analyse similaire du groupe Renault montre qu'il est encore moins utilisé en proportion, quelle que soit l'audience visée par le discours. Ce constat semble indiquer que les entreprises ne s'appuient pas clairement aujourd hui sur l'environnement pour se présenter comme des acteurs sociaux

\section{LÉGITIMITÉ COGNITIVE : CONVAINCRE DE SON ENGAGE- MENT EN LE PRESENTANT COMME EVIDENT}

Moins explicite que les précédentes stratégies discursives, la communication qui repose sur la recherche d'une légitimité cognitive informe sans plus d'explications que PSA développe des labels environnementaux internes (comme Airdream) ou applique des labels externes (ex : la production dans des usines certifiées ISO14001), conclut des partenariats avec des organismes qui défendent l'environnement, collabore avec des institutions scientificues, obtient des récompenses pour son engagement environnemental, possède une position dominante sur le marché des véhicules propres, a recours à des technologies, sources d'énergies ou pratiques considérées comme "propres » (ex : filtre a particules, moteur hybride, biodiescl, recyclage), etc.

Les occasions d'affirmer son engagement vis-a-vis des différentes parties prenantes sont nombreuses. Lorsque l'on s'adresse aux consommateurs par l'intermédiaire de publicités, la communication visuelle s'ajoute au langage : l'utilisation d'images (grands espaces), de couleurs (vert / bleu) et d'icônes symbolisant l'écologie (ex : l'icône de la technologie HDI est un bouquet de fleurs vertes) permettent également de conforter PSA dans son image d'entreprise citoyenne sans avoir recours au discours. Cette stratégie a pour avantage de capter l'attention dans un espace de communication réduit (comme dans la presse magazine). Enfin, ce type de strattégie discursive permet de convaincre la cible que performance et écologie ne sont pas antinomicues, ce qui prouve aussi son utilité clans la communication financière.
De par son caractère affirmatif et la diversité des thènes qu'il permet d'aborder, le message qui légitime l'action environnementale de manière cognitive semble constituer une stratégie discursive à la fois simple (il se passe de justification) et incontournable pour l'ensemble des cibles de l'organisation, comme le montrent les exemples présentés dans le tableau 3.

\section{CONCLUSION}

Les questions environnementales font aujourd'hui partie de la stratégie des entreprises et des marques, quel que soit leur niveau dimplication dans la dégradation de l'environnement. Dans un secteur considéré comme particulièrement polluant, PSA, au-delà de ses actions en faveur de l'environnement, doit relever le défi de la communication. En effet, défendre sa réputation auprès de ses différentes cibles passe autant par la communication que par l'action (9). Pour relever ce défi environnemental, PSA déploie des stratégies discursives reposant sur l'utilisation de toutes les formes de légitimité pour chacune des cibles, mais avec des intensités et des tonalités différentes. De manière générale, autant la communication pragmatique semble particulièrement adaptée aux consommateurs et aux actionnaires, autant jouer sur les valeurs morales semble davantage approprié lorsqu'il s'agit des employés et des consommateurs. La palette cognitive, de par sa variété, peut quant à elle, aisément sadapter aux différentes attentes des trois parties prenantes.

S'agissant de la légitimité pragmatique, PSA démontre sa capacité à jouer sur un discours basé à la fois sur le registre des coûts et sur celui de la performance financière. Le groupe indique clairement au consommateur que choisir un véhicule respectueux de l'environnement, cest gagner financièrement en économisant de l'essence sans pour autant empiéter sur son plaisir de conduire. PSA se différencie ainsi de Renault en réussissant à décliner les bénéfices clients liés aux voitures "vertes " beaucoup plus intensément et de surcroit en utilisant une palette de messages plus variée. PSA parvient d'une part à montrer à ses employés que sa politique environnementale est source d'économies (d'énergie, de matières premières etc.) et d'autre part a ses actionnaires qu'investir sur l'environnement est également une source d'avantage concurrentiel et de performance financière. Cet aspect est confirmé par Peter Karandysovsky, Responsable de la Communication Développement Durable chez PSA Peugeot Citroën. 
qui rappelle que "le développement durable constitue aujourd'hui la meilleure solution à la crise financière actuelle qui affecte le secteur automobile ² $^{2}$.

Chez PSA, comme la plupart de ses concurrents, la légitimité morale est sous employée dans le discours de la marque. Il est cependant dommage de s'en priver, car ce type de stratégie discursive permet de s'adresser à deux des cibles évoquées sous un registre identitaire valorisant. Concernant les consommateurs, le discours moral contribue en effet à un sentiment d'autonomic, synonyme de différenciation sur la base de la consommation vertueuse : consommer "vert ", c'est consommer "bien ».Concemant les employés, le discours moral joue sur la carte de l'affiliation qui se traduit, d'une part par le renforcement du sentiment d'appartenance au groupe, et d'autre part par la fierté de faire partie d'unce entreprise respectueuse de l'environnement. La légitimité morale est quasi absente des rapports annuels de PSA, comme nous l'avons signalé. La communication aux actionnaires gagnerait cependant à utiliser cette stratégie. En effet, se présenter comme unc entreprise vertueuse permet également de renforcer le capital marque, et par conséquent la valeur du groupe.

La palette cognitjve, quant à elle, est très utilisée par PSA comme par ses principaux concurrents. Ce type de stratégie discursive peut prendre de multiples formes. Loin de constituer un simple complément aux autres stratégies discursives, elle semble au contraire en constituer le socle de par son adaptation aussi bien au message verbal qu'au message iconique, et sied donc particulièrement bien à la communication publicitaire, où le message environnemental se traduit facilement en images et symboles. Si PSA et l'ensemble de l'industric automobile ont bien compris son intérêt, le recours a ce type de stratégie peut s'avérer délicat. Le pas entre communication éco-responsable et campagne de greenuwshing qui désigne toute communication reposant sur une utilisation abusive de l'argument écologique $-(4,10)$ est en effet vite franchi. Notons que les récentes recommandations de l'ARPP' devraient contribuer à limiter ce risque dans la communication à destination des consommateurs.

\section{Notes}

1 Cette étude porte sur l'analyse de plusieurs vecteurs de communication du groupe PSA entre 2006 et 2009 : les journaux internes (destinés aux employés), les rapports annuels (destinés aux actionnaires et investisseurs), et les publicités publiées dans 11 titres de la presse magazine (destinées aux consommateurs).

2. Interview réalisée par les deux auteurs en décembre 2009 .
3 Les recommandations de l'Autorité de Régulation Professionnelle de la Publicité (ARPP) peuvent être consultées sur http://www.arpp-pub.org/Nouvelle-Recommandation. html

\section{Références}

(1) Balmer J.M.T. (2001). Corporate branding. corporate identity. and corporate marketing. Seeing through the fog. European Journal of Marketing, 35 (3-4), 248-291.

(2) Bansal P. et Clelland, 1. (2004), Talking taash: Legitimacy, impression management, and unsystematic risk in the context of the natural environment. Academy of Management Journal, 47.93-103.

(3) Béji-Bécheur A. et Bensebaa F. (2009), Les stratégies de positionnement responsable : le cas des tours opérateurs, Décisions Marketing. 54, avril-juin, 39-49.

(4) Benoit-Moreau F. Larceneux F. et Parguel B. (2010), La communication sociétale : entre opportunités et risque d'opportunisme. Décisions Markéting. 59. juillet-septembre. 75-78.

(5) Fournier S. (1998). Consumers and their brands: Developing relationship theory in consumer research, Journal of Consumer Research, 24, 343-373.

(6) Gioia D.A. et Chittipeddi, K. (1991). Sensemaking and sensegiving in strategic change initiation, Strategic Management Joumal, 12, 433-448.

(7) Hatch M.J. et Schultz M. (2003), Bringing the corporation into corporate branding. European Journal of Marketing. 37 (7-8), 1041-1064.

(8) Lacoeuilhe J. (1997), Le concept d'attachement ì la marque dans la formation du comportement de fidélité. Revie Française du Marketing, 165, 5. 29-42.

(9) Philippe D. (2009), Perceptions sociales et structuration des marchés : trois essais sur l’impact des stratégies de conformité et framing sur le statut, la réputation et lat légitimité des organisations. Thèse de doctorat en Sciences de Gestion. HEC Paris.

(10) Philippe D. et Durand R. (à paraître). The impact of norm-conforming behaviors on firm reputation, Strategic Management Journal.

(11) PSA Peugeol Citroën (2008), Rapport d'activité et de développement durable.

(12) Suchman M.C. (1995), Managing legitimacy: strategic and institutional approaches, Academy of Management Review. 20 (3). 571-610. 